\title{
MAGNETIC BRAIDING DUE TO WEAK ASYMMETRY
}

A, B, RECHESTER AND $T, H$, STIX

\section{PLASMA PHYSICS LABORATORY}

\section{MASTER}

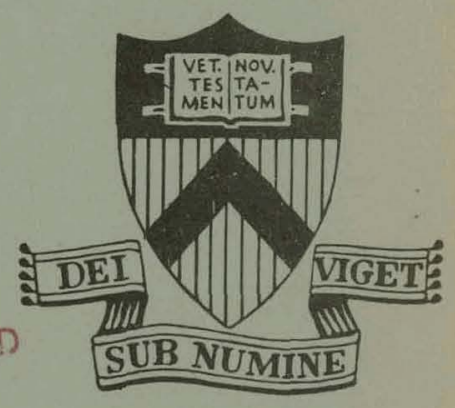

\section{PRINCETON UNIVERSITY PRINCETON, NEW JERSEY}

This work was supported by U. S. Energy Research and Development Administration Contract E(11-1)-3073. Reproduction, translation, publication, use and disposal, in whole or in part, by or for the United States Government is permitted. 


\section{DISCLAIMER}

This report was prepared as an account of work sponsored by an agency of the United States Government. Neither the United States Government nor any agency Thereof, nor any of their employees, makes any warranty, express or implied, or assumes any legal liability or responsibility for the accuracy, completeness, or usefulness of any information, apparatus, product, or process disclosed, or represents that its use would not infringe privately owned rights. Reference herein to any specific commercial product, process, or service by trade name, trademark, manufacturer, or otherwise does not necessarily constitute or imply its endorsement, recommendation, or favoring by the United States Government or any agency thereof. The views and opinions of authors expressed herein do not necessarily state or reflect those of the United States Government or any agency thereof. 


\section{DISCLAIMER}

Portions of this document may be illegible in electronic image products. Images are produced from the best available original document. 
NOTICE

This report was prepared as an account of work sponsored by the United States Government. Neither the United States nor the untted SLales Encrgy Researrh and Development Administration, nor any of their employees, nor any of their contractors, subcontractors, or their employees, makes any warranty, express or implied, or assumes any legal liability or responsibility for the accuracy, completeness or usefulness of any information, apparatus, product or process disclosed, or represents that its use would not infringe privately owned rights.

Printed in the United States of America.

Available from

National Technical Information Service

U. S. Department of Commerce 5285 port Royal Road

Springfield, Virginia 22151

Price: Printed Copy $\$ \star * ;$ Microfiche $\$ 1.45$

NTIS

*Pages

$1-50$

$51-150$

$151-325$

$326-500$

501-1000
Selling Price

$\$ 4.00$

5.45

7.60

10.60

13.60 
Magnetic Braiding due to Weak Asymmetry*

Alexander B. Rechester and Thomas H. Stix Plasma Physics Laboratory, Princeton University Princeton, New Jersey 08540

Magnetic surfaces for a plasma with a helical current perturbation $\sim \varepsilon^{2}$ are destroyed by toroidal effects or by a second current perturbation, of incommensurate helicity, and the behavior of magnetic field lines becomes stochastic in layers of relative

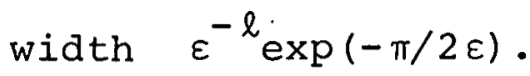

This work considers how the magnetic surfaces for a tokamak discharge are affected by helical perturbations of the plasma current. Such current perturbations are known to be associated with resistive modes, 1,2 and substantial experimental evidence has been offered ${ }^{3}$ for the occurrence of magnetic islands 4 associated with nonlinear tearing instabilities. 5,6 In this paper we show that the existence of two such modes with different helicity or the effect of toroidal geometry on a single such mode leads to the destruction of magnetic surfaces. The resultant stochastic wandering or "braiding" of the magnetic lines can produce collisionless radial heat transport, enhanced current penetration and radial particle transport, and may change the inductance for toroidal plasma current flow so that sudden onset of braiding would produce negative or positive spikes in the loop-voltage signal. 7

The mechanism of magnetic surface destruction ${ }^{8}$ was first investigated in two classic papers on magnetic irregularities, 9,10 
where it was demons'trated that a spectrum of overlapping resonances produces 'stochastic wandering of the magnetic field lines. In this work we start with a field of helical symmetry and exact magnetic surfaces which exhibits a single set of primary islands, i.e., a single resonance at some $r=r_{0}$ between the helical variation and the rotational transform $l(r)$. Weak asymmetry is introduced via a first-order magnetic perturbation of different helicity which might be due to toroidal effects or to the presence of a second magnetic resonance at $r=r_{1}$. The incommensurate perturbation is found to produce little secondary islands which appear wherever the Fourier components of the perturbation resonate with the local transform, $\omega(k)$, Eq. (4), within the primary islands. In fact, a pile-up occurs with a denumerable infinity of chains of these little secondary islands appearing as the primary separatrix is approached from both inside and outside. Calculating the width of the secondary islands in each such chain and the spacing between adjacent chains, we find that the relative thickness of the braiding region, within which secondary-island overlap occurs (see Fig. 1), is given by a non-analytic function of the expansion parameter, $\varepsilon$, namely $\varepsilon^{-\ell} \exp (-\pi / 2 \varepsilon)$. $\varepsilon$, defined in Eq. (8), is proportional to the thickness of the primary Islands and is inversely proportional to $m_{1}\left|r_{1}-r_{0}\right| / m . \quad \ell \simeq 2\left|m_{1} / m\right|+1$, and $\dot{m}$ and $\mathrm{m}_{1}$ are azimuthal mode numbers for the original helical field and for the perturbation. This result may be compared with an earlier estimate $^{11}$ for the thickness of the stochastic layer $\sim \exp (-1 / \varepsilon)$. We also find that toroidal effects produce stochastic regions around the separatrices associated with any magnetic island. As 
a specific illustration, we consider the $m=2$ ST tokamak islands, measured by von Goeler, ${ }^{3}$ and evaluate the fraction of island area in which the magnetic field is stochastic.

It is worth noting that the results obtained here may be directly applied also to the analysis of the motion of a charged particle in the field of two incommensurate plane waves. ${ }^{11}$

We consider, in zero order, a magnetic field of helical symmetry, $\vec{B}(r, u), u \equiv m \theta-n z / R . r, \theta$, and $z$ are the usual cylindrical coordinates. The field is derivable from a vector pontential, $\overrightarrow{\mathrm{B}}=\nabla \times \overrightarrow{\mathrm{A}}(r, u)$, and dịrect substitution will verify that the flux function ${ }^{12} \psi=\mathrm{mA}_{\mathrm{z}}+\mathrm{n}(\mathrm{r} / \mathrm{R}) \mathrm{A}_{\theta}=-\int\left[\mathrm{mB}_{\theta}-(\mathrm{nr} / \mathrm{R}) \mathrm{B}_{\mathrm{z}}\right] \mathrm{dr}$ satisfies the condition for a magnetic surface, $\vec{B} \cdot \nabla \psi=0$. We define $2 \pi / l(r) \equiv q(r) \equiv r<B_{z}>/ R<B_{\theta}>$, where the averages are taken over a period in $u$, and, making use of the identity $r B_{r}=\partial \psi / \partial u$, expand around the rational surface $r=r_{0}$, at which $n q\left(r_{0}\right)-m=0$. With $B_{r}=b(r)$ sin $u$, which we might imagine stems from a helical ripple in the plasma current distribution equivalent to $\Delta j_{\eta} \sim \delta\left(r-r_{0}\right) \cos u$, we find

$$
\begin{aligned}
& \psi \simeq-\left(\frac{m}{2 \pi} \frac{r<B_{z}>}{R} \frac{d_{l}}{d r}\right)_{r=r_{0}} \xi, \\
& \xi \equiv(1 / 2)\left(r-r_{0}\right)^{2}-\Gamma \cos u \\
& \Gamma \equiv\left[-(2 \pi b R) /\left(m<B_{z}>d_{l} / d r\right)\right]_{r=r_{0}},
\end{aligned}
$$

The island separatrix is given by the $\xi=\gamma^{2}$ contour, $\gamma \equiv|\Gamma|^{1 / 2}$, and the full width of the islands is $4 \gamma$. See Fig. 1. 
$: r$

Introducing orthogonal coordinates $\xi, \alpha, \eta$ which are local to the primary islands, the coordinate $\xi$, Eq. (1), parameterizes the magnetic surfaces and $\nabla \xi$ will be everywhere perpendicular to them. The third coordinate, $\eta$, is chosen so that $\nabla n$ is directed along the $r=$ constant, $u=$ constant helical lines and is normalized so that $|\nabla \eta|=1$. $\hat{\alpha}$ is perpendicular to $\hat{n}$ and to $\hat{\xi}, \hat{\alpha}=\hat{n} \times \hat{\xi}$, and we choose to calibrate $\alpha$ so that its rate of change along $\eta$ is a Iunclion of $\xi$ alnne, $i . e .$, so that $\dot{\alpha}=d \alpha / d n \sim \omega(\xi)$, with the important advantage that Eq. (6), below, will then be immediately soluble by simple Fourier analysis.

One may verify that the choice $d \alpha / d u=(d \alpha / d n) /(d u / d n)=$ $\gamma(\partial \xi / \partial r)^{-1}$ will provide that $\omega \sim d \alpha / d \eta$ remains constant along a line of force, i.e., on a single $\xi$ surface. We now introduce new variables, $k(\xi)$,

$$
0 \leqslant k^{2} \equiv\left(\gamma^{2}+\xi\right) / 2 \gamma^{2},
$$

and, inside the separatrix, $\zeta(k, u)$, such that $k \sin \zeta \equiv \sin (u / 2)$. The integration of $\mathrm{d} \alpha / \mathrm{du}=\gamma(\mathrm{d} \xi / \mathrm{dr})^{-1}$ then leads directly to $\zeta=\operatorname{am}(\alpha, k)$ and the coordinate transformation equations, $u=2 \sin ^{-1}[k \operatorname{sn}(\alpha, k)]$ and $r-r_{0}=2 \gamma k \operatorname{cn}(\alpha, k)$, where sn and $c n$ are Jacobian elliptic functions, periodic with the period $4 \mathrm{~K}(\mathrm{k})$, and where $\mathrm{K}(\mathrm{k})$ is the complete elliptic intcgral of the first kind. The angle variable $\alpha$ increases by the increment $4 \mathrm{~K}(\mathrm{k})$ on one complete circuit (see Fig. 1); if we renormalize this increment to $2 \pi$, then the effective rotational transform in the island interior, relative to the local magnetic axis (the elliptic stagnation point), will be $\omega(k)$, 


$$
\omega(k)=\frac{2 \pi L}{4 K(k)} \frac{d \alpha}{d \eta}=\frac{\pi m}{2} \frac{\gamma}{k(k)} \frac{d l}{d r},
$$

where $L \equiv 2 \pi R\left[1+\left(n r_{0} / m R\right)^{2}\right]^{l / 2}$, the distance along $\left\langle\vec{B}\left(r_{\circ}, u\right)\right\rangle$ for $\Delta z=2 \pi R$.

Distances between nearby contours $\xi$ and $\xi+\mathrm{d} \xi$ are related by $|\mathrm{d} \vec{r}|=\mathrm{d} \xi|\nabla \xi|^{-1}=2 \gamma\left[\mathrm{cn}^{2} \alpha+\left(\mathrm{m} \gamma / \mathrm{r}_{0}\right)^{2} \mathrm{sn}^{2} \alpha \mathrm{dn}^{2} \alpha\right]^{-1 / 2} \mathrm{dk}$, and the area inside a closed contour $\xi(\mathrm{k}), \mathrm{k} \leqslant 1$, is given by

$$
A_{i}=\frac{16 \gamma k\left(1-k^{2}\right)}{|\nabla u|} \frac{d k(k)}{d k}
$$

where $|\nabla \mathrm{u}|=\mathrm{mL} / 2 \pi r_{0} \mathrm{R}$. $\mathrm{A}_{\mathrm{i}}$ may also be considered the action variable conjugate to the angle variable $\alpha$; the Ilamiltonian would be $\xi\left(A_{i}\right)$. Outside the separatrix, the appropriate substitution leads to $u=2 a m[\beta,(1 / k)], r-r_{0}=2 \gamma k d n[\beta,(1 / k)]$, and in (4), $\mathrm{K} \rightarrow \mathrm{K}(1 / \mathrm{k})$. See Fig. 1 . Distances are related by $|d \vec{r}|=2 \gamma\left[\mathrm{dn}^{2} \beta+\left(\gamma \mathrm{m} / \mathrm{kr}_{0}\right)^{2} \operatorname{sn}^{2} \beta \mathrm{cn}^{2} \beta\right]^{-1 / 2} \mathrm{dk}$, and the total area between the upper and lower $\xi(k), k \geqslant 1$, contours from $\beta=0$ to $2 \mathrm{~K}(1 / \mathrm{k})$ is $\mathrm{A}_{\mathrm{O}}=16 \mathrm{k}|\nabla \mathrm{u}|^{-1} \mathrm{E}(1 / \mathrm{k})$, where $\mathrm{E}$ is the complete elliptic integral of the second kind.

We now introduce weak asymmetry through the first-order perturbation, $\mathrm{B}_{r}{ }^{(1)}=\mathrm{b}^{(1)} \sin \mathrm{w}, \mathrm{b}^{(1)}=$ constant, as miqht be induced by helical plasma current ripples at an adjacent surface, $r=r_{1}$. The first step is Fourier analysis of the perturbation field, $\sim F^{(1)}, \overline{E q} .(6)$, into harmonics of the variabies $\alpha$ and $n$. We define $\lambda$ and $k$ such that on $r=r_{0}, w=m_{1} \theta-n_{1}(z / R)=$ $\lambda u-k(n / R), \lambda \simeq m_{1} / m, k \simeq n_{1}-\left(m_{1} n / m\right)$. The error in the approximate forms for $\lambda$ and $k$. is of order $\left(r_{0} / q R\right)^{2}$. In the 
ensuing Fourier analysis, $2 \lambda$ is required to be integral, but the results should be representative of the analysis for other values of $\lambda \cdot$ The new magnetic surfaces will, near $r=r_{0}$, be of the form $\phi^{(0)}(k)+\phi^{(1)}(k, \alpha, n)=$ constant, and will satisfy the first-order equation, $\vec{B}^{(0)} \cdot \nabla \phi(1)=\left(\hat{n}_{n}+\hat{\alpha}_{\alpha}\right) \cdot \nabla \phi(1)=$ $-\overrightarrow{\mathrm{B}}^{(1)} \cdot \nabla \phi(0)$. We approximate $B_{\eta}$ as constant, and use Eq. (4) together with $\mathrm{d} \alpha / \mathrm{d} \eta=|\nabla \alpha| \mathrm{B}_{\alpha} /|\nabla \eta| \mathrm{B}_{\eta}$ to evaluate $|\nabla \alpha| \mathrm{B}_{\alpha}$, to find, Inside the separatrix,

$$
\begin{aligned}
& L \frac{\partial \psi}{\partial \eta} \frac{(1)}{n}+\frac{4 K(k)}{2 \pi} \omega(k) \frac{\partial \phi(1)}{\partial \alpha}=F^{(1)} \frac{d \phi(U)}{d \xi}(\xi) \\
& F^{(1)} \equiv-L \vec{B}^{(1)}(w) \cdot \nabla \xi / B_{n}
\end{aligned}
$$

Although Eq. (6) was derived for thin primary islands, its use of the action $\left[\mathrm{A}_{i}(\mathrm{k})\right.$ or $\left.\mathrm{A}_{\mathrm{O}}(\mathrm{k})\right]$ - angle $(\alpha)$ formalism assures us that an equation of this same form could be obtained for thick primary islands.

To solve Eq. (6), we Fourier-expand hath ${ }^{(1)}$ and $F^{(1)}$,

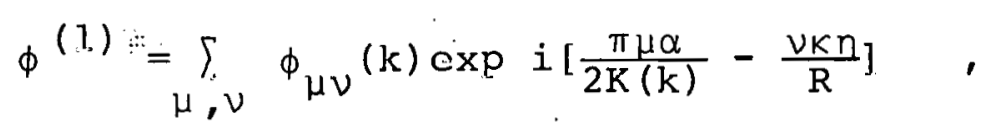

and substitute to obtain $\phi_{\mu \nu}(k)=-i F_{\mu \nu}(k)(d \phi(0) / d \xi) \cdot$ $[\mu \omega(k)-2 \pi \nu k]^{-1}$, using $L \simeq 2 \pi R$. Since we are free to choose the function $\phi^{(0)}(\mathrm{k})$, the simplest selection for solubility is $\mathrm{d} \phi(0) / \mathrm{dk}=\pi_{\mu, \nu}\left(\mathrm{k}-\mathrm{k}_{\mu \nu}\right)$ where $\omega\left(k_{\mu \nu}\right)=2 \pi \nu k / \mu$ for each $\mu, \nu$. Then the magnetic surfaces can be determined by expanding $\phi^{(0)}+\phi^{(I)}=$ constant around each little resonance, $\mathrm{k}=\mathrm{k}_{\mu \nu}$,

$$
\xi_{k} \simeq \frac{\left(k-k \nu^{i}\right)^{2}}{2}-i\left(\mu \frac{d \omega}{d k} \frac{d \xi}{d k}\right)^{-1} \sum_{ \pm} F_{\mu \nu} \exp i\left(\frac{\pi \mu \alpha}{2 k(k)}-\frac{\nu k n}{R}\right)
$$


summing just over $\mu, \nu$ and $-\mu ;-\nu$.

To determine $\mathrm{F}_{\mu \nu}$,

$F_{\mu \nu}(k)=[8 \pi K(k)]^{-1} \int_{0}^{2 \pi} d(n K / R) \int_{0}^{4 K} d \alpha F(w, k) \exp i[-(\pi \mu \alpha / 2 K)+(\nu K n / R)]$,

with $F(w, k) \approx-L\left(b(1) / B_{n}\right)\left(r-r_{0}\right) \sin [\lambda u-(k n / R)]$, we recall

$r-r_{0}=2 \gamma \mathrm{kcn}(\alpha, \mathrm{k}), \lambda \simeq \mathrm{m}_{1} / \mathrm{m}$, and $\mathrm{u} / 2 \simeq \sin ^{-1}(\mathrm{k} \operatorname{sn} \alpha) \equiv \Omega$.

Then we expand, for example for $2 \lambda$ even, $\sin (\lambda u)=\sin (2 \lambda \Omega)$

$=(-1)^{\lambda+1} 2^{2 \lambda-1} \cos \Omega \sin ^{2 \lambda-1} \Omega+\ldots=(-1)^{\lambda+1} 2^{2 \lambda-1} \mathrm{dn} \alpha(k \operatorname{sn} \alpha)^{2 \lambda-1}+\ldots$

and introduce similar expansions for $2 \lambda$ odd and for $\cos \left(2 \mathrm{~m}_{1} \Omega / \mathrm{m}\right)$.

Contributions to $F_{\mu \nu}$ from subsequent terms in the series are

algebraically small in the expansion parameter, $\varepsilon$, Eq. (8). The $\alpha$-integral is easily evaluated if its contour is depressed, for $\mu>0$, to run from $(0,0)$ to. $(0,-i \infty)$ to $(4 \mathrm{~K},-i \infty)$ to $(4 \mathrm{~K}, 0)$.

Expanding the integrand about the uppermost pair of singular points,,$^{13}$ we use, for example near $\alpha=2 K-i K^{\prime}, K^{\prime} \equiv K\left[\left(1-k^{2}\right)^{1 / 2}\right]$, $\mathrm{cn} \alpha=\mathrm{cn}\left(2 \mathrm{~K}-\mathrm{i} \mathrm{K}^{\prime}+\Delta \alpha\right)=\operatorname{dn}(\Delta \alpha)[i \mathrm{k} \operatorname{sn}(\Delta \alpha)]^{-1}$, etc., with the expansion parameter $\varepsilon$,

$$
\varepsilon \equiv\left|\frac{m_{\gamma}\left(\mathrm{d}_{1} / \mathrm{dr} r\right)}{2 \pi k}\right| \approx\left|\frac{\mathrm{m}_{\gamma}}{m_{1}\left(r_{1}-r_{0}\right)}\right| \text {. }
$$

(The evaluation of $F_{\mu \nu}$ can be carried through in a straightforward fashion without the small-e expansion -- e.g., picking up all the residues, etc. -- but the result is cumbersone.) The argument for $\omega$ and for the $K$ and $K^{\prime}$ elliptic integrals is $\mathrm{k}_{\mu \nu}$; and $v= \pm 1 \cdot \mathrm{r}_{1}$ is the radius at which $q\left(r_{1}\right)$ would eyual $m_{1} / n_{1}$ it $d_{l} / d r$ were constant. In this manner we find $\mathrm{F}_{\mu \nu}$ for $\mu>0$, and for $\mu<0$ we use $\mathrm{F}_{-\mu,-\nu}=\mathrm{F}_{\mu \nu}{ }^{*}$. Then Eq. (7) takes the standard form for island contours, 


$$
\begin{aligned}
\xi_{k} & =\frac{\left(k-k_{\mu \nu}\right)^{2}}{2}-\Gamma_{k} H \cos \left(\frac{\alpha}{\varepsilon}-\frac{|\omega k| \eta}{\omega R}\right) \\
\Gamma_{k} & \equiv \frac{2^{2 \lambda-2} \pi}{(2 \lambda) !} \frac{b^{(I)}}{b} \frac{1}{k(d K / d k)} \frac{\exp \left(-k^{\prime} / \varepsilon\right)}{\varepsilon^{2 \lambda-1}} .
\end{aligned}
$$

where $\mathrm{H} \equiv[\operatorname{Sgn}(\omega K)]^{\mu}$. The full width of the little islands in. terms of $k$ i.s $4 \gamma_{k}, \quad \gamma_{k} \equiv\left|\Gamma_{k}\right|^{1 / 2}$. From the resonance condition $\omega\left(k_{1, v)}\right)=2 \pi \nu k / \mu$ and using $|\cdot \nu|=1$, we can find the increment in $k_{\mu \nu}, \Delta \mathrm{k}$, corresponding to adjacent resonant surfaces determined by $|\Delta \mu|=1$. Using (4) and (8), $\Delta \mathrm{k}=2 \pi|\mathrm{k}|\left[\mu^{2} \mathrm{~d} \omega / \mathrm{dk}\right]^{-1}=\pi \varepsilon[2 \mathrm{dK} / \mathrm{dk}]^{-1}$. Because $\omega(\mathrm{k}) \rightarrow 0$ as $k \rightarrow 1$, Eq. (4), resonances will occur for all integer $\mu>\mu_{\min }$. (A similar pile-up also occurs as the separatrix is approached from the outside.) overlap of little islands will begin when $\Delta \mathrm{k}=4 \gamma_{\mathrm{k}}$, which occurs when

$$
\sigma \equiv \frac{k}{2 d K / d k}=\frac{2^{2 \lambda+3}}{\pi(2 \lambda) ! \cdot} \frac{b^{(1)}}{\dot{b}} \frac{\exp \left(-K^{\prime} / \varepsilon\right)}{\varepsilon^{2 \lambda+1}}
$$

For $k \rightarrow 1, k(2 d k / d k)^{-1} \approx 1-k=\delta k$ and $k^{\prime}(k) \simeq \pi / 2$. As noted before, the thickness, $\delta \mathrm{k}$, of the stochastic layer is given by a non-analytic function of $\varepsilon$, namely, $\sim \varepsilon^{-2 \lambda-1} \exp (-\pi / 2 \varepsilon)$. For fixed $b, \varepsilon$ varies as $\left(d_{l} / d r\right)^{1 / 2}$ and in this circumstance high-shear regions will be especially susceptible to magnetic braiding. Of some interest also is the steep rise of the function $\varepsilon^{-2 \lambda-1} \exp \left(-K^{\prime} / \varepsilon\right)$ prior to reaching its maximum at $\varepsilon=K^{\prime} /(2 \lambda+1)$ : In this range a modest increase in $\varepsilon$ can produce a sudden large increase in the thickness of the braiding layer.

Outside of the zero-order separatrix, the expressions for 
$\omega$ and $\varepsilon$ are the same as before except that $K=K(1 / k)$, etc. Equations for the new magnetic surfaces in the neighborhood of $\mathrm{k}=\mathrm{k}_{\mu \nu}$ are in the form of set (9) but with $\alpha \rightarrow \beta$, $(\mathrm{kaK} / \mathrm{dk})^{-1} \rightarrow-\mathrm{k}^{2 \lambda}[\mathrm{dK}(1 / \mathrm{k}) / \mathrm{dk}]^{-1}$ and $\mathrm{H} \rightarrow(1 / 2)\left[1+(-1)^{\mu+2 \lambda}\right]$. $[1+\operatorname{sgn}(\omega k)]$. Resonances appear only for $\operatorname{sgn}(\omega k)>0$ and $|\Delta \mu|=2$, so that the density of little islands and the thickness of the braiding layer are reduced to approximately half their inside-separatrix values. The expression for the overlap condition resembles (10) except that the new left-hand side reads $\left[\mathrm{k}^{2 \lambda} \mathrm{dK}(1 / \mathrm{k}) / \mathrm{dk}\right]^{-1} \sim 2 \delta \mathrm{k}$.

Using the results of the previous paragraphs together with the expression for $A_{i}$, Eq. (5), and for $A_{0}$, we can easily derive an expression for the ratio of the total stochastic area both inside and outside the primary separatrix, $\delta \mathrm{A}$, to the total area, A, within the primary separatrix, valid for $\sigma \ll 1$,

$$
\delta A / A \simeq(0.75 \sigma) \ln (27.4 / \sigma) \quad
$$

The considerations here can be applied to study the effect of toroidal geometry on magnetic islands. To first order in $r / R$, the axisymmetric poloidal and toroidal fields are given ${ }^{14}$ by $B_{p}(\psi)[1+(r / R) \Lambda(\psi) \cos \theta]$ and $B_{t}(\psi)[1-(r / R) \cos \theta]$; the quantity $\Lambda+l$ is proportional to the magnitude of the effective "vertical" field needed to balance the well-known toroidal hoop forces. We compute the excitation function $F=\pi \gamma^{2} n(\Lambda+1)$. $(r / K)\{\sin [(m+l) \theta-n z / R]+\sin [(m-1) \theta-n z / R]\}$, and carry out the perturbation analysis as before to find, with $m_{1}=m \pm 1$, $\mathrm{n}_{1}=\mathrm{n}$, 


$$
\frac{\mathrm{k}}{2 \mathrm{dK} / \mathrm{dk}}=\left|\frac{m r(\Lambda+1)}{\pi \mathrm{R}}\right| \frac{2^{2 \lambda+1}}{(2 \lambda-1) !} \frac{\exp \left(-\mathrm{K}^{\prime} / \varepsilon\right)}{\varepsilon^{2 \lambda+1}}
$$

as a measure of the beginning of little-island overlap and magnetic braiding just inside the separatrix.

Abel inversions of $\mathrm{x}$-ray intensity oscillations from the ST tokamak indicated, 9 just prior to disruption, the presence of a magnetic island near the $q=2$ surface of $4 \gamma=3.5-4 \mathrm{~cm}$ full thickness. Üsing $\mathrm{m}=2, \mathrm{n} \equiv 1, \mathrm{r}_{\mathrm{o}}-8 \mathrm{~cm}, \mathrm{k}-109 \mathrm{~cm}$, $\mathrm{d} . / \mathrm{dr}=0.4 \mathrm{~cm}^{-1}$, and thus $\varepsilon=0.24$ and $\lambda=3 / 2$, and setting $\Lambda=0$, simultaneous solution of Eqs. (5) and (12) shows that $\sim 30 \%$ of the interior ared of the $m=2$. island would be braided by the toroidal perturbation of the symmetry, close to the maximum of (12) with.respect to variation of $\varepsilon$.

\section{ACKNOWLEDGMENTS}

It is a pleasure to acknowledge the help of Drs. S. Cohen, J. Ehrman, J. Finn, M. N. Rosenbluth, S. von Goeler, and the useful comments of the referees.

*This work was supported by the United States Energy Research and Development Administration (formerly Atomic Energy Commission), Contract $\mathrm{E}(11-1) 3073$. 


\section{REFERENCES}

${ }^{1}$. P. Furth, J. Killeen, and M. N. Rosenbluth, Phys. Fluids 6, 459 (1963) .

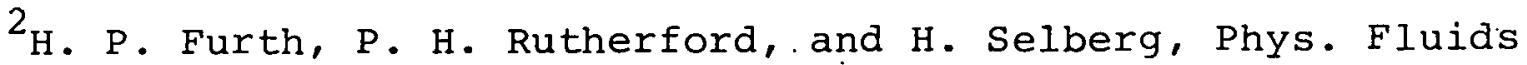
16, 1054 (1973).

${ }^{3}$ s. von Goeler, Seventh Eur. Conf. on Controlled Fusion and Plasma Physics, Lausanne (1975).

${ }^{4}$ D. W. Kerst, J. Nucl. Energy, Part C 4 , 253 (1962).

${ }^{5}$ P. H. Rutherford, Phys. Fluids 16, 1903 (1973).

${ }^{6}$ B. V. Waddell, D. A. Monticello, M. N. Rosenbluth, and R. B. White, Bull. Am. Phys. Soc. 20, 1342 (1975).

${ }^{7}$ T. H. Stix, Phys. Rev. Lett. $\underline{30}, 833$ (1973).

${ }^{8}$ H. Grad, Phys. Fluids 10, 137 (1967).

9. N. Rosenbluth, R. Z. Sagdeev, J. B. Taylor, and G. M. Zaslavski, Nucl. Fusion $\underline{6}, 297$ (1966).

10 N. N. Filonenko, R. Z. Sagdeev, and G. M. Zaslavsky, Nucl. Fusion ㄱ, 253 (1967).

${ }^{11}$ G. M. Zaslavskii and N. N. Filonenko, Sov. Phys. JETP $\underline{27}$, 851 (1968). See also G. M. Zaslavskii and B. V. Chirikov, Sov. Phys. Uspekhi 14, 549 (1972).

$12 \mathrm{~J}$. L. Johnson, C. R. Oberman, R. M. Kulsrud, and E. A. Frieman, Phys, Fluids 1, 201 (1998).

${ }^{13}$ See, for example, E. Jahnke and F. Emde, Tables of Functions, 4 th edition (Dover, New York, 1945), Chap. VI. 
i $\quad$ i

${ }^{1.4} \mathrm{~V}$. D. Shäfronov, in Reviews of Plasma Physics, edited by M. A. Leontovich (Consultants Bureau, New York, 1966).

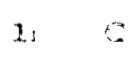

i. 5 


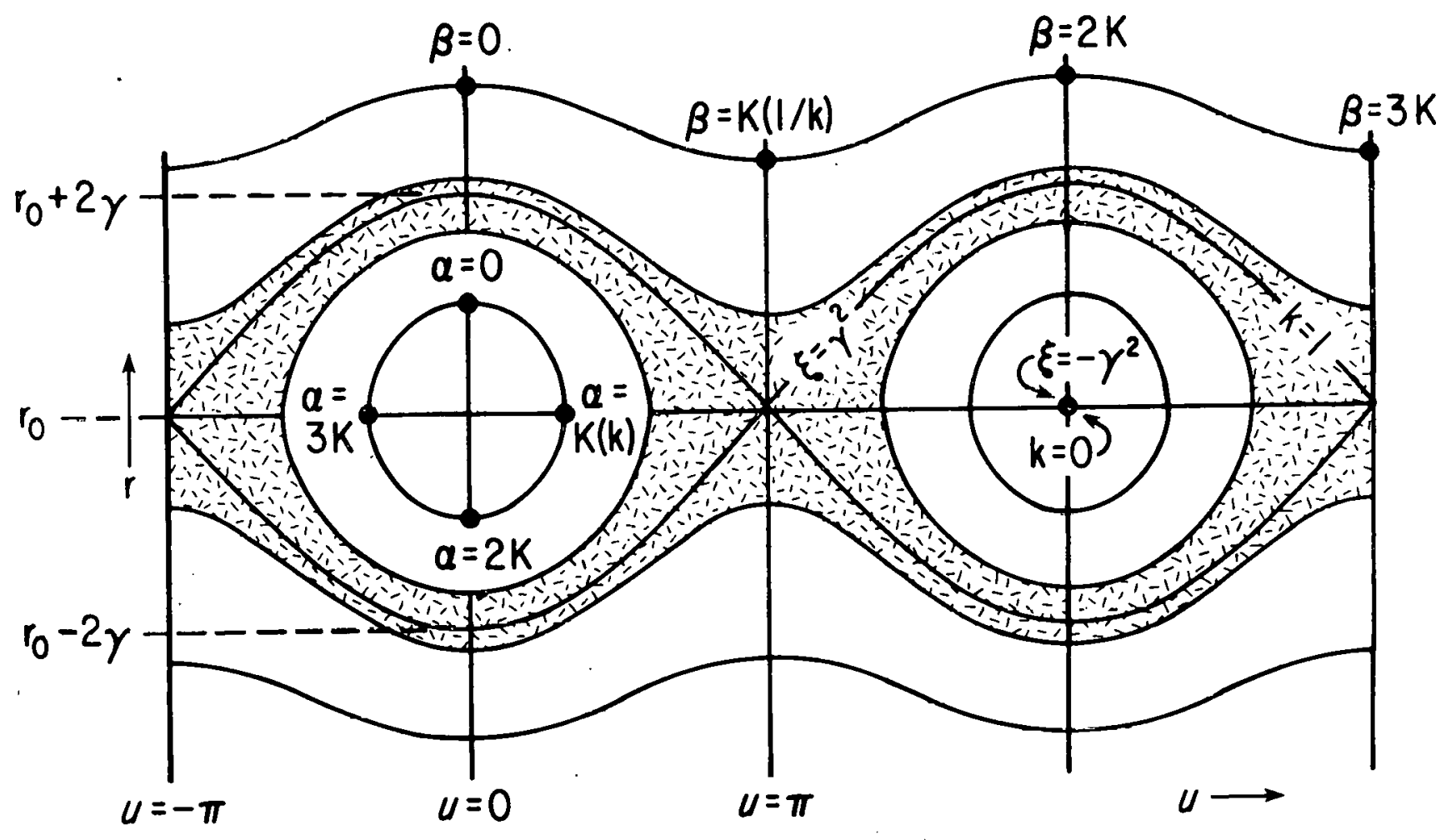

753982

Fig. 1. Structure of primary magnetic islands in the vicinity of $r=r$, showing several $\xi=$ constant contours and, at selected points, Values of the angle variables $u, \alpha$ and $\beta . \Gamma>0$. Shading indicates a representative region of "braiding," $i, e .$, stochasticity. 